\title{
Cartographie De La Sensibilite Aux Maladies Environnementales Respiratoires Dans Le District Sanitaire De Koumassi-Port-Bouët-Vridi (Sud De La Côte d'Ivoire)
}

\author{
Kouame Adonis Krou Damien \\ Fofana Kandana Eve Maïmouna \\ Mobio Abaka Brice Hervé \\ Kassi Ahon Jean-Baptiste \\ Kouame Koffi Fernand \\ Djagoua Eric M'moi Valère \\ Université Félix HOUPHOUET-BOIGNY/ \\ UFR des Sciences de la Terre et des Ressources Minières/ \\ Centre Universitaire de Recherche et d'Application en Télédétection \\ (CURAT), Abidjan, Côte d'Ivoire
}

doi: 10.19044/esj.2017.v13n5p202 URL:http://dx.doi.org/10.19044/esj.2017.v13n5p202

\begin{abstract}
In Côte d'Ivoire morbidity causes are related to diseases such as malaria, diarrhea, and acute respiratory infections (ARI), which are constantly evolving. Koumasi and Port-Bouet are the most affected municipalities in the country.

This study aims to find environmental determinants of spatial distribution of the ARIs and identify the most sensitive areas to take action for prevention and effective control and targeted in order to reduce the prevalence of ARI.

To achieve this goal, we have identified environmental factors associated with the occurrence of ARI. Different environmental and cartographic data were integrated into a GIS. A multi-criteria analysis was performed to determine the environmental parameters that expose more people to the occurrence of the IRA and the weights of these parameters. The combination of exposure maps was used to map the sensitivity to IRA.

Multi-criteria analysis revealed that refuse dumps have the highest influence in the occurrence of the disease, followed by industrial facilities and permanent wastewater. The ARI sensitivity map obtained shows that neighborhoods Nord-est 2 and Zone industrielle in Koumassi municipality are most susceptible to ARI. In the municipality of Port-Bouet sensitivity is
\end{abstract}


more pronounced at Vridi canal and Vridi 3. Special attention should therefore be granted these neighborhoods.

Keywords: Geographic Information System, environmental factors, multicriteria analysis, Acute Respiratory Infections, Abidjan

\section{Résumé}

En Côte d’Ivoire les causes de morbidité sont liées aux maladies telles que le paludisme, la diarrhée, et les infections respiratoires aiguës (IRA) qui sont en évolution constante. Koumassi et Port-Bouët sont les communes les plus touchées par les IRA dans le pays (Ministère de la santé et de la lutte contre le sida (2014).

La présente étude se propose de trouver les déterminants environnementaux de la répartition spatiale des IRA et d'identifier les zones les plus sensibles pour mener des actions de prévention et de lutte efficaces et ciblées en vue de réduire la prévalence des IRA.

Pour atteindre cet objectif, nous avons identifié les facteurs environnementaux associés à la survenance des IRA dans la zone d'étude. Les différentes données environnementales et cartographiques recueillies ont été intégrées dans un SIG. Une analyse multicritère a été réalisée en vue de déterminer les paramètres environnementaux exposant le plus les populations à la survenance des IRA et leurs poids. La combinaison des cartes d'exposition a permis de cartographier la sensibilité aux IRA.

L’analyse multicritère a révélé que les dépôts d’ordures ont le niveau d’influence le plus élevé dans la survenance de la maladie, suivis des unités industrielles et des eaux usées permanentes. La carte de sensibilité aux IRA obtenue montre que les quartiers Nord-est 2 et Zone industrielle dans la commune de Koumassi sont les plus sensibles aux IRA. Dans la commune de Port-Bouët la sensibilité est plus prononcée à Vridi canal et Vridi 3. Une attention particulière devrait donc être accordée à ces quartiers.

Mots-clés: Système d'Information Géographique, facteurs environnementaux, analyse multicritères, Infections Respiratoires Aiguës, Abidjan

\section{Introduction}

La santé d’une population est déterminée par une multitude de facteurs sociaux, économiques et environnementaux (Tran, 2004). Malheureusement elle est compromise par diverses maladies. Les plus récurrentes en Afrique et dans les pays en développement sont le paludisme, les infections respiratoires aiguës (IRA), le choléra, la tuberculose et les maladies diarrhéiques pour ne citer que ces quelques cas. Elles sévissent et 
causent beaucoup de dégâts dans plusieurs communautés et constituent un frein au développement (Tran, 2004).

En Côte d'Ivoire, les IRA représentent après le paludisme, la seconde cause d'hospitalisation et de mortalité, notamment chez les enfants de moins de 5 ans (MSLS, 2014). En effet, l'on enregistre une incidence des IRA qui varie de 16 à 79 \%. Localement cette incidence atteint par endroit $200 \%$ comme c'est le cas dans le district de KPV (RASS, 2013).

Les IRA sont le résultat de plusieurs facteurs imbriqués. Une simple description des différences de mortalité et de morbidité isolée de ses manifestations cliniques dans la population ne suffit pas pour mettre en place des politiques et programmes adéquats de lutte contre cette maladie (Tchatchou, 2012). Il est donc important de les traiter de façon groupée afin de déceler dans le cadre de vie les zones les plus vulnérables à cette pathologie, pour ainsi connaître les espaces prioritaires en cas d'intervention.

Les connaissances sur la morbidité, les conditions sociales et environnementales sont nécessaires pour expliquer les interactions entre les IRA et l'environnement. Les facteurs environnementaux et l'état de santé ont un point commun : ils s'articulent tous deux dans l'espace et sur le territoire et peuvent dans ce cas être analysés et gérés à l'aide des outils de la géomatique (Bénié et al., 2000). Ces outils permettent la cartographie de la distribution de la maladie pour la surveillance épidémiologique, la mise en place de systèmes d'alerte et de cartes de risque et la mise en évidence de relation entre la maladie et l'environnement.

La présente étude a pour objectif d’identifier les déterminants de la distribution spatiale des IRA dans le district sanitaire de Koumassi-PortBouët-Vridi (KPV) dans l'optique de cartographier la sensibilité aux IRA. Cela pourrait servir d'outil d'aide à la décision aux autorités administratives et sanitaires pour mener des actions de prévention et de lutte efficaces et ciblées, en vue de réduire la prévalence des IRA.

\section{Présentation de la zone de l'étude}

\section{- Situation géographique de la zone d'étude}

Le district sanitaire de Koumassi-Port-Bouët-Vridi est situé dans la ville d'Abidjan en Côte d'Ivoire. Il s'étend sur une superficie de $125 \mathrm{Km}^{2}$. Il est limité à l'Ouest par les communes de Treichville et Marcory, à l'Est par la ville de Grand-Bassam. Il est situé entre les Latitudes $3^{\circ} 50^{\prime}$ et $4^{\circ} 40^{\prime}$ Ouest et les Longitudes $5^{\circ} 14^{\prime}$ et $5^{\circ} 20^{\prime}$ Nord et est composé des communes de Koumassi et Port-Bouët (figure 1).

La commune de Koumassi est située entre $5^{\circ} 17^{\prime} \mathrm{N}$ et $3^{\circ} 59^{\prime} \mathrm{O}$ et s'étend sur une superficie de $13,65 \mathrm{~km}^{2}$ soit 2,4\% de la ville d'Abidjan. Elle comprend quatorze quartiers où l'habitat est implanté sur 65\% de sa surface. 
La commune de Port-Bouët quant à elle s'étend sur une superficie de $111,1 \mathrm{~km}^{2}$. Elle est localisée entre $\underline{5}^{\circ} 20^{\prime} \mathrm{N}$ et $4^{\circ} 00^{\prime} \mathrm{O}$. La commune de PortBouët est divisée en trois secteurs géographiques regroupant 45 comités de base ou quartiers : Port-Bouët centre, Port-Bouët route de Bassam et PortBouët Vridi.

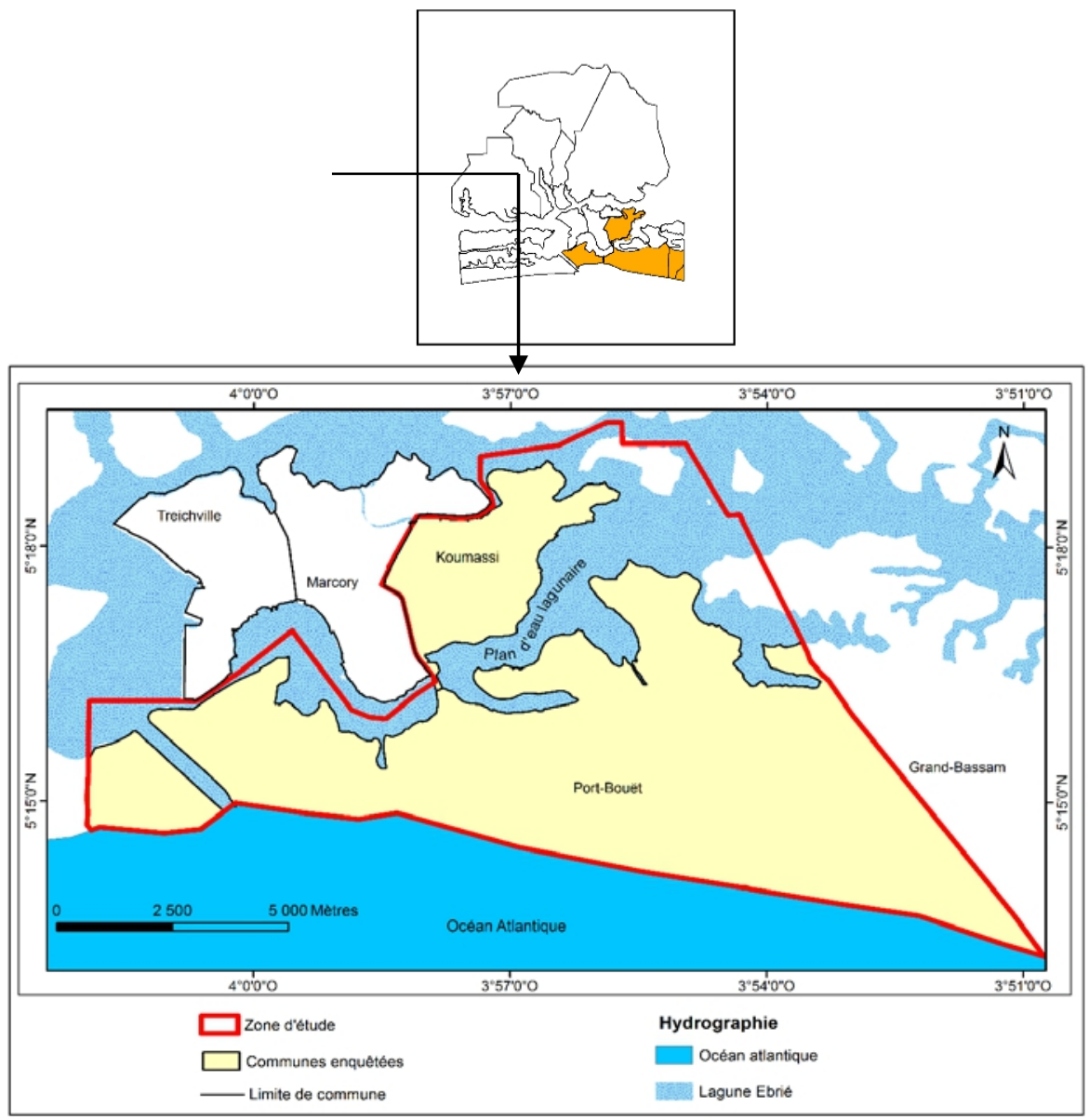

Figure 11: Présentation du District sanitaire de Koumassi-Port-Bouët-Vridi

\section{- $\quad$ Caractéristiques physiques et humaines de la zone d'étude}

\section{Caractéristiques physiques}

Le climat de la zone d'étude est de type subéquatorial chaud et humide comportant une grande saison des pluies (Mai-Juin-Juillet), une petite saison des pluies (Septembre-Novembre) et deux saisons sèches. La grande saison sèche débute en Décembre et prend fin en Mars. Cette variation saisonnière influence fortement la décomposition des ordures et entraine le processus de fermentation des déchets (Soro et al, 2010), ce qui a des répercussions immédiates sur la qualité de l’air. 
La hauteur moyenne annuelle des précipitations déterminée sur la période 1963-2000 est de 1700 mm (Kouamé, 2007) in (Soro et al., 2010). La température est presque toujours aux environs de $27^{\circ} \mathrm{C}$ et le degré d'hygrométrie annuel moyen est supérieur à 80\% (SODEXAM).

Sur un relief composé essentiellement de plaine, le sol est de type sablonneux avec des marécages par endroits. Au niveau hydrographique, le district sanitaire est drainé par la lagune Ebrié et bordé au sud de la commune de Port-Bouët par l'océan Atlantique comme l'indique la figure 1.

\section{Caractéristiques humaines}

La population de notre espace d'étude a connu une évolution constante de 1975 à 2015 avec une population qui est passée de 225000 à 82500 habitants entre ces deux dates.

En ce qui concerne la densité de population, en 2014 celle de Koumassi est estimée à $51227 \mathrm{hbts} / \mathrm{km}^{2}$ sur un espace de 13,65 km², contre 3402 hbts/km² pour Port-Bouët sur un espace de 111,1 km² (INS, 2015).

Au niveau des secteurs d'activités, la population des deux communes est à majorité active dans le secteur tertiaire (commerce, activités informelles...) suivie du secteur secondaire (activités industrielles) puis du secteur primaire. Aussi, il faut noter qu'à Port-Bouët au moins $10 \%$ de la population pratique l'agriculture, il s'agit pour l'essentiel du maraichage, notamment la culture de salade et de tomate (INS, 2015).

\section{Matériel et méthodes}

\section{- Matériel}

Les données utilisées pour la réalisation de cette étude sont d'ordre cartographique, épidémiologique et environnemental.

\section{Les données cartographiques}

Il s'agit d'une part de la carte du découpage administratif des communes de Koumassi et Port-Bouët par quartier à l'échelle 1 : 50 000, datant de 2010, acquise auprès du Centre de Cartographique et de Télédétection du Bureau National d'Etude Technique et de Développement (CCT/BNETD) ; et d'autre part de la carte du district sanitaire de KoumassiPort-Bouët-Vridi à l'échelle 1 : 95000 datant de 2013 qui a été obtenue à partir de la carte sanitaire de la DIPE/MSLS. Toutes ces données sont dans le référentiel géodésique WGS 84 et projetées en UTM zone $30 \mathrm{~N}$.

\section{Les données épidémiologiques}

Elles ont été collectées auprès des responsables du district sanitaire et portent sur l'évolution du nombre de cas d'IRA enregistré par centre de santé. Ces données nous apportent des informations sur le malade (âge, sexe, 
lieu de résidence). Elles seront également confrontées à celles issues de l’enquête ménage.

\section{Les données environnementales}

Elles sont relatives aux facteurs environnementaux permettant d'appréhender l'exposition aux risques d'IRA et susceptibles d'altérer la qualité de l'air, car les IRA sont intimement liées à l’air que nous respirons. Nous avons ainsi pu recenser à l'aide de GPS les unités industrielles, les dépôts sauvages d'ordures et les eaux usées permanentes qui se trouvaient être les facteurs les plus pertinents dans notre espace d'étude.

Le logiciel de cartographie ArcGis 10.2.1 a servi à l'extraction des données du GPS, à l'analyse spatiale (mise en relation des données sémantiques et géométriques), et à la restitution cartographique.

\section{- Méthodes}

Evaluation multicritère des facteurs environnementaux de risque d'IRA

Nous avons effectué une classification de ces paramètres et un poids a été attribué à chacun d'eux en fonction de leur niveau d'influence dans l'altération de la qualité de l'air et donc dans la survenance des IRA chez les populations. Elle est réalisée à partir du Processus de Hiérarchie Analytique qui est basée sur le modèle de pondération d'additif (Banai 1993, Wu 1998, Basnet et al. 2001, Zhu and Dale 2001). Cela a l'avantage de permettre de calculer les coefficients de pondération associés aux attributs de couches cartographiques (Chakhar, 2006). Cette analyse s’est basée sur la méthode d'agrégation complète. A travers un calcul des ratios celle-ci nous a permis d’appréhender le facteur qui influe fortement sur la qualité de l’air dans le district sanitaire.

Par la suite, la Méthode de Hiérarchie Multicritère (MHM) développée par Saaty (1984) nous a permis d'attribuer un poids à chaque critère pour ainsi effectuer des cartes de sensibilité. Pour ce faire, une matrice a été construite, suivie de l’établissement des différentes priorités.

La construction de la matrice passe par l'attribution des valeurs numériques qui se fait à partir des jugements sur l'importance d'un facteur par rapport à un autre.

La détermination des vecteurs de priorité commence par la sommation de chaque colonne. Ensuite, on procède à la normalisation de la matrice qui consiste à diviser chaque valeur d'appréciation d'une colonne par la somme de cette colonne.

Enfin on effectue le calcul de la moyenne de chaque ligne afin d'obtenir le poids de chaque facteur dans l'état morbide des populations face aux IRA 
L'évaluation de la cohérence de notre analyse a consisté au calcul de l'Indice de Cohérence (IC) et du Ratio de Cohérence (RC). Un raisonnement est dit cohérent lorsque l'IC et le RC sont inférieurs ou égaux à 10\% (Alla, 2013). Ils s’obtiennent par les calculs suivants :

$$
\begin{aligned}
& I C=\frac{x m o y-\text { nombre de colonne }}{\text { nombre decolonnes }-1} \\
& \mathrm{RC}=\frac{\mathrm{IC}}{\mathrm{IA}} \quad \text { (2) }
\end{aligned}
$$

Où: $\chi$ moy est la moyenne des résultats obtenus et IA est l'indice aléatoire (égal à 0,58 pour 3 critères).

La moyenne des résultats s'obtient à la suite des étapes ci-dessous :

- $\quad$ Multiplication des appréciations de chaque colonne par le poids lui correspondant.

- $\quad$ Sommation des valeurs de chaque ligne ;

- Division de chaque total de ligne par la priorité du facteur correspondant à cette ligne ;

- $\quad$ Détermination de la moyenne des résultats ;

\section{Détermination du niveau d'exposition lié aux paramètres environnementaux}

La survenance des IRA au sein de la population résulte de la combinaison des facteurs environnementaux, sociaux, démographiques et économiques (Tchatchou, 2012 ; Aubry, 2014). En effet, le manque d'accès à l'eau potable, la pollution de l'air (intérieur et extérieur), la mauvaise évacuation des déchets ménagers et le manque d'hygiène alimentaire augmenteraient les risques sanitaires chez l'enfant (Woldemicael, 2000; Dongo et al., 2008; Mesbah, 2009). Pour ce qui est du district sanitaire de Koumassi-Port-Bouët-Vridi, nous avons enregistré trois critères pertinents liés à l'environnement immédiat des populations, pouvant altérer la qualité de l'air. Il s'agit des unités industrielles, des eaux usées permanentes et des dépôts d'ordures.

Les différents critères recensés ont fait l'objet d'une interpolation à partir de l'algorithme IDW du module analyse spatiale sous ArcGis. Cela nous a permis d'obtenir les cartes d'exposition aux IRA pour chacun des critères.

\section{Cartographie de la sensibilité aux IRA}

Pour la réalisation de la carte de sensibilité aux IRA, nous avons effectué une pondération des critères déterminés. Celle-ci a consisté à affecter à chaque facteur de risque d'IRA son coefficient de pondération pour tenir compte de l'importance qu'on lui accorde dans le processus de pollution de l'air. La formule suivante a été utilisée

Sensibilité $=\mathrm{E} 1 * \mathrm{P} 1+\mathrm{E} 2 * \mathrm{P} 2+\mathrm{E} 3 * \mathrm{P} 3$ 
Avec P1, 2, 3 = poids des critères 1, 2, 3 et E1, 2, 3 = attributs des cartes d'exposition 1, 2, 3

\section{Résultats et discussion}

\section{- Résultats}

Analyse multicritère des facteurs environnementaux de risque d'IRA

Le calcul des ratios nous a permis de connaître le facteur qui influe fortement sur la qualité de l'air dans le district sanitaire à travers une classification des critères (tableau I).

Tableau I: Classification des critères selon le produit des ratios

\begin{tabular}{|c|c|c|c|c|}
\hline & Koumassi & Port-Bouët & Produit des ratios & Classification \\
\hline Unités industrielles & 0,28813559 & 0,357142857 & 0,102905569 & $2^{\text {eme }}$ \\
\hline Eaux usées permanentes & 0,49152542 & 0,339285714 & 0,166767554 & $1^{\text {er }}$ \\
\hline Dépôts d'ordures & 0,22033898 & 0,303571429 & 0,06688862 & $3^{\text {ème }}$ \\
\hline
\end{tabular}

Les valeurs numériques attribuées à partir des jugements sur l'importance d'un facteur par rapport à un autre sont présentées dans le tableau II.

Tableau II: Comparaison binaire des facteurs de pollution

\begin{tabular}{|c|c|c|c|}
\hline & Eaux usées permanentes & Unités industrielles & Dépôts d'ordures \\
\hline Eaux usées permanentes & 1 & 0,33 & 0,14 \\
\hline Unités industrielles & 3 & 1 & 0,2 \\
\hline Dépôts d'ordures & 7 & 5 & 1 \\
\hline Total & 11 & 6,33 & 1,34 \\
\hline
\end{tabular}

Les poids obtenus pour chacun des facteurs dans l'état morbide des populations face aux

IRA sont présentés dans le tableau III.

Tableau III : Vecteurs de priorité et poids des différents facteurs

\begin{tabular}{|c|c|c|c|c|c|}
\hline & $\begin{array}{c}\text { Eaux usées } \\
\text { permanentes }\end{array}$ & $\begin{array}{c}\text { Unités } \\
\text { industrielles }\end{array}$ & $\begin{array}{c}\text { Dépôts } \\
\text { d'ordures }\end{array}$ & Somme & $\begin{array}{c}\text { Poid } \\
\text { s }\end{array}$ \\
\hline $\begin{array}{c}\text { Sommation des colonnes } \\
\text { (comparaison binaire) }\end{array}$ & 11 & 6,33 & 1,34 & & \\
\hline Eaux usées permanentes & 0,09 & 0,05 & 0,10 & 0,24 & 0,08 \\
\hline Unités industrielles & 0,27 & 0,16 & 0,15 & 0,58 & 0,19 \\
\hline Dépôts d'ordures & 0,64 & 0,79 & 0,75 & 2,18 & 0,73 \\
\hline & & & & $\begin{array}{c}\text { Somme } \\
\text { poids }\end{array}$ & 1 \\
\hline
\end{tabular}

Au niveau de l’évaluation de la cohérence de notre analyse a permis d’obtenir comme moyenne des résultats 3,04.

En définitive, nous obtenons en nous basant sur les équations (1) et

(2) les résultats suivants ; IC = $2 \%$ et $\mathbf{R C}=\mathbf{3 , 4 5} \%$.

IC et $\mathrm{RC}<10 \%$, donc le raisonnement utilisé est cohérent.

Cartographie des zones d'exposition aux IRA 
Après interpolation des principaux critères impliqués dans l'altération de la qualité de l'air au niveau du district sanitaire de KPV, nous avons pu obtenir des cartes d'expositions représentées sur les figures 2, 3 et 4.

La liste des quartiers concernés par l'étude et numérotés de 1 à 13 identifiés sur les différentes cartes est dressée dans le tableau IV

Tableau IIV : Liste des quartiers de l'espace de l'étude

\begin{tabular}{|c|c|}
\hline $\mathrm{N}^{\circ}$ & Quartier \\
\hline 1 & Sicogi 1 \\
\hline 2 & Zone industrielle \\
\hline 3 & Sopim pangolin \\
\hline 4 & Nord-est 1 \\
\hline 5 & Nord-est 2 \\
\hline 6 & Gonzagueville \\
\hline 7 & Adjouffou 1 \\
\hline 8 & Derrière wharf \\
\hline 9 & Phare littoral \\
\hline 10 & Abattoir 3 \\
\hline 11 & Petit Bassam \\
\hline 12 & Vridi canal \\
\hline 13 & Vridi 3 foyers \\
\hline
\end{tabular}

La figure 2 présente les zones d'exposition due aux unités industrielles. A Koumassi tout comme à Port-Bouët, les espaces les plus exposés sont les quartiers qui renferment les activités industrielles. Il s'agit du quartier zone industrielle à Koumassi, Vridi 3 foyers et Vridi canal dans la commune de Port-Bouët. Dans les autres quartiers qui ont fait l'objet de l'enquête, nous n'avons pas recensé d'unité industrielle, ce qui explique la faible exposition dans ces espaces.

La figure 3 met en relief les zones d'exposition dues aux eaux usées permanentes. Hormis quelques endroits où on les rencontre de manière isolée, les eaux usées sont plus concentrées dans les quartiers bordés par la lagune et à proximité des zones industrielles (Nord-est 1, Nord-est 2, zone industrielle à Koumassi, et Vridi 3 foyers à Port-Bouët). Cela exprime aussi la qualité de l'environnement sanitaire dans le district sanitaire. 


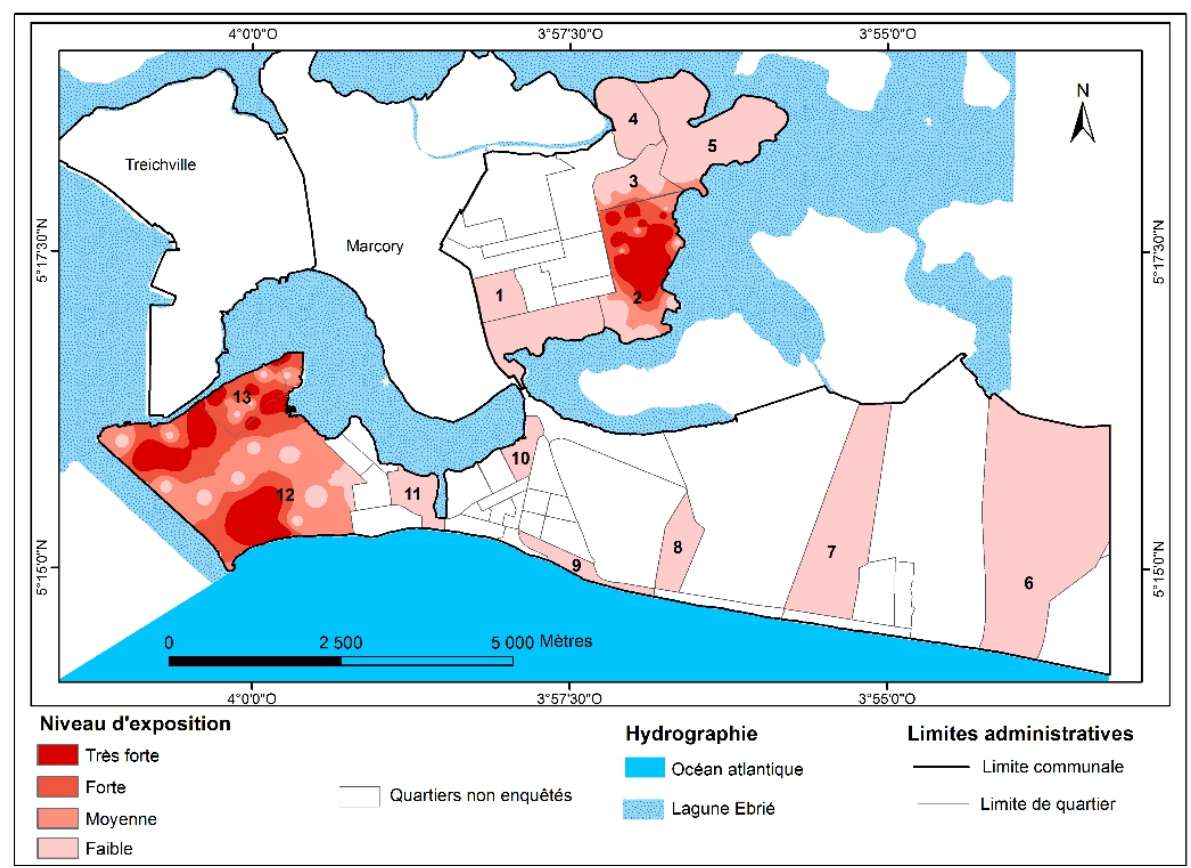

Figure 2 : Carte d'exposition due aux unités industrielles

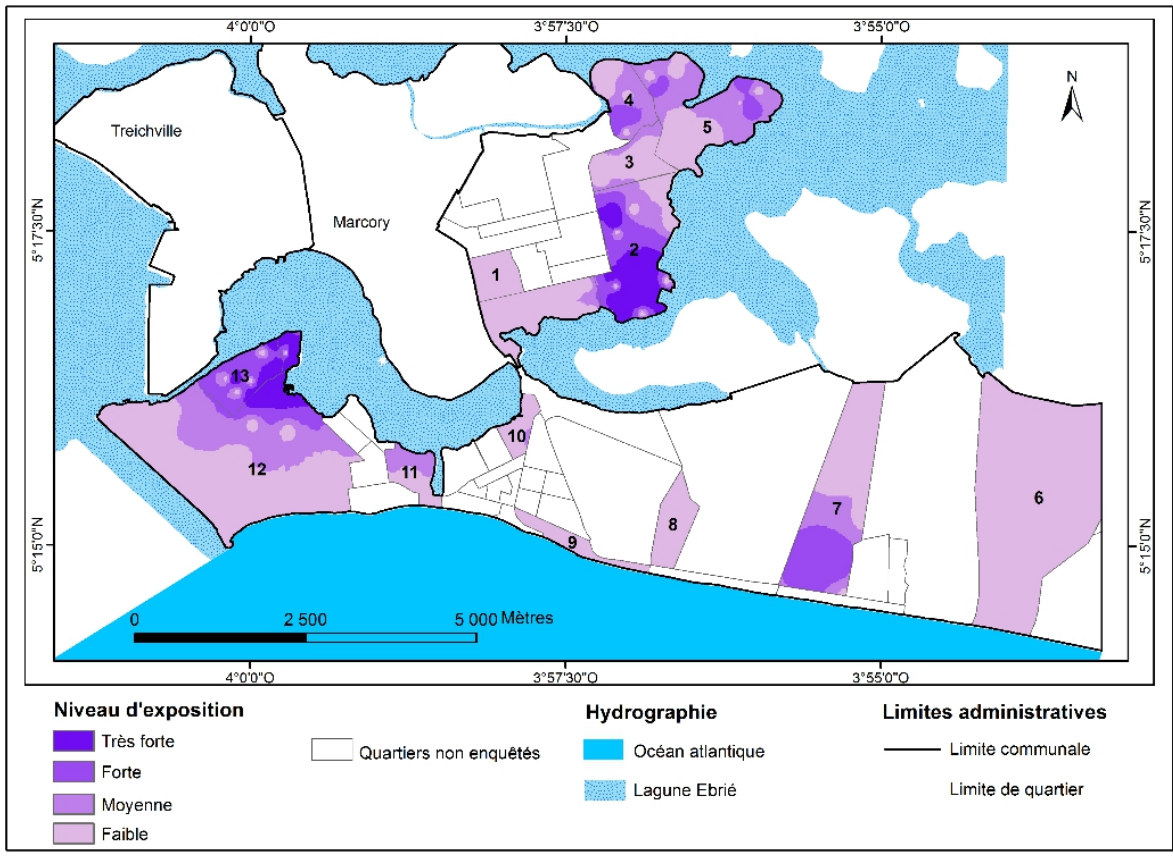

Figure 3 : Carte d'exposition due aux eaux usées permanentes

La figure 4 présente l'exposition liée aux dépôts d'ordures solides qui dégagent des odeurs pestilentielles. Dans la commune de Koumassi l'exposition due aux eaux usées est très forte au quartier Nord-est 2 et forte 
dans la zone industrielle. Dans la commune de Port-Bouët la très forte exposition est marquée au niveau de Vridi 3 foyers et de l'Abattoir 3. On remarque par ailleurs que les zones de très forte exposition sont bordées par la lagune. En effet l'humidité liée à la présence de l'eau accentue la décomposition des ordures et dégage de ce fait des odeurs à la fois nauséabondes et piquantes, avec des répercussions sur le système respiratoire des populations riveraines.

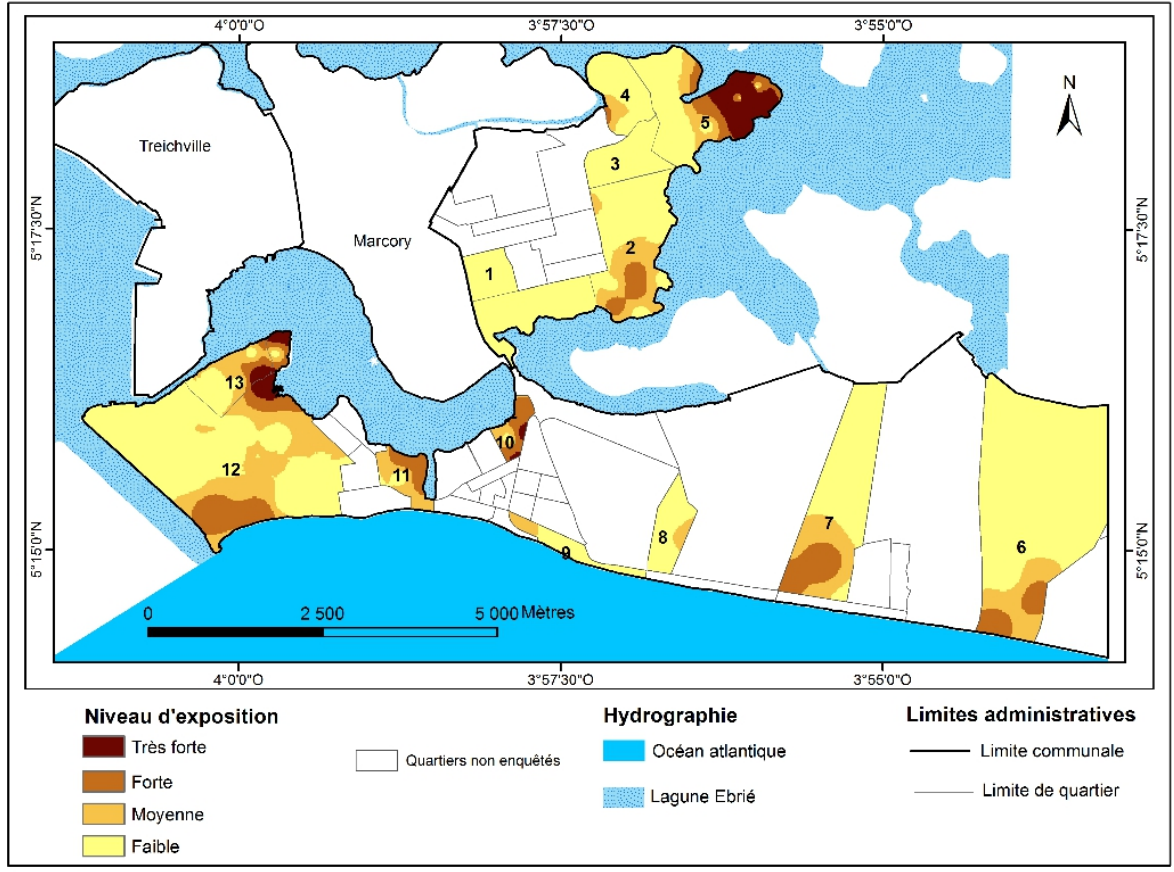

Figure 4 : Carte d'exposition due aux dépôts d'ordures

\section{Cartographie des zones de sensibilité aux IRA}

La pondération des facteurs d'altération de la qualité de l'air nous a permis d'établir une carte de sensibilité aux IRA, présentée sur la figure 5.

Deux zones de forte sensibilité sont présentent dans la commune de Koumassi. Il s'agit des quartiers zone industrielle et Nord-est 2. Dans les quartiers Nord-est 1, Sopim pangolin et Sicogi 1. A part quelques endroits peu sensibles, la majorité de ces espaces est faiblement sensible à la maladie.

En ce qui concerne la commune de Port-Bouët, la forte sensibilité est observée au niveau des quartiers Vridi 3 foyers et Vridi canal. Ainsi, les zones jugées à sensibilité moyenne ou faible traduisent un risque d'exposition moins important, voire inexistant par rapport aux facteurs environnementaux déterminés. 


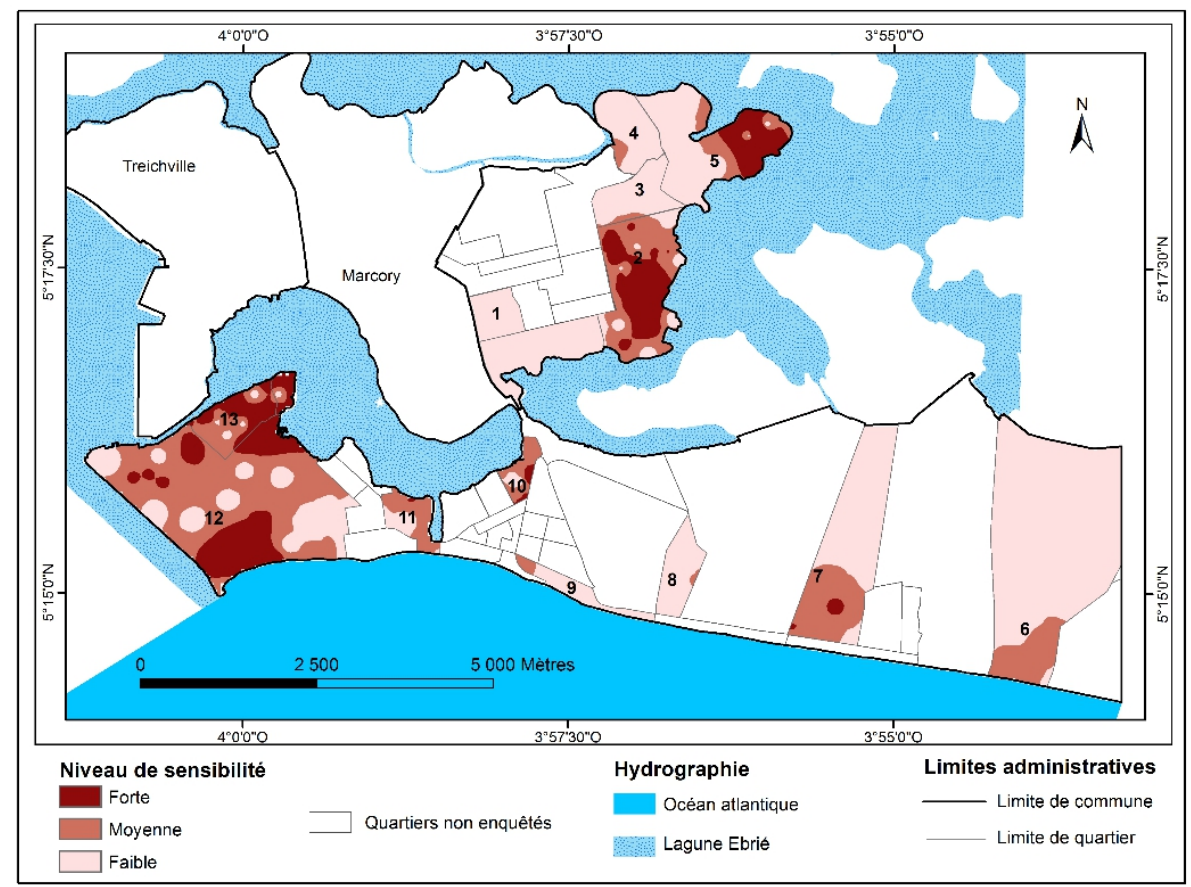

Figure 5 : Carte de sensibilité aux IRA

\section{Discussion}

Le principe d'élaboration des différentes cartes s'est basé sur l'analyse spatiale, à partir de différents critères déterminés qui se rapportent à l'environnement immédiat des populations dans les zones enquêtées. Cette cartographie nous a permis de présenter distinctement les espaces géographiques les plus sensibles aux infections respiratoires aiguës.

Dans chacune des deux communes, deux principales zones de forte sensibilité ont été décelées, il s'agit pour l'essentiel des quartiers incluant des zones industrielles. Cependant nos résultats montrent que certains des quartiers situés hors des zones de forte sensibilité présentent une proportion importante de cas d'IRA.

Les résultats obtenus au niveau du district sanitaire nous montrent que les zones de forte sensibilité sont celles où les prévalences sont les plus élevées (Vridi canal et Vridi 3 foyers dans la commune de Port-Bouët, avec 97,5\% ainsi que Nord-Est 2 et Zone industrielle dans la commune de Koumassi avec 76,5\% et 69\%). Cela montre la fiabilité de la carte de sensibilité obtenue.

Trois des quatre quartiers déterminés à forte sensibilité contiennent des zones industrielles. Ces résultats sont en rapport avec ceux de Dongo et al, (2013) qui montre que les pollutions engendrées par les industries à Abidjan sont majoritairement de natures atmosphérique (68\%) et hydrique (30\%). Pour lui les pollutions hydriques ont pour conséquences des 
manifestations atmosphériques car ces eaux usées dégagent des odeurs nauséabondes. L’occurrence des IRA étant étroitement liée à la qualité de l'air, cela justifie la très forte prévalence de 97,5\% d'IRA aux abords des zones industrielles d’où les fortes sensibilités observé dans les quartiers situés à la périphérie de cette zone.

Les sensibilités élevées dans la zone d’étude sont dues à la forte pollution urbaine enregistrée en Afrique subsaharienne. Les multiples explications de la détérioration du cadre de vie font courir aux populations vivant en milieu urbain un grand risque de morbidité par infections respiratoires aiguës comme cela a été montré par Tchatchou, (2012).

Au niveau de la voirie, le district sanitaire de Koumassi-Port-BouëtVridi possède un important boulevard (boulevard VGE) et de nombreuses voies principales très empruntées. La proximité de ces voies avec les habitations peut donc être considérée comme un facteur d'exposition aux IRA. En effet, plusieurs études (Airparif, 2012 ; Branchu et al., 2013) ont montré que la présence d'axes routiers fortement empruntés à proximité des habitations est un véritable facteur de pollution de l'air, qui est responsable de la création et l'accentuation de bon nombre de maladies respiratoires. Nos résultats obtenus au niveau du quartier Sicogi 1 Koumassi montre en effet que, ce dernier est situé dans une zone de faible sensibilité, mais possède un niveau de prévalence de 64,06\%. Cette prévalence élevée pourrait s'expliquer par la densité du réseau routier dans ce quartier. La non prise en compte du réseau routier pourrait être une limite dans ce travail. Toutefois, c'est la combinaison de plusieurs facteurs qui donne la sensibilité aux IRA et le quartier Sicogi 1 est le seul à se trouver dans cette situation de prévalence élevée dans une zone de faible sensibilité, nous pouvons dire que nos résultats sont acceptables.

\section{Conclusion}

L’utilisation du système d’information géographique couplée à l'analyse multicritères nous a permis de montrer que les différents quartiers du district ne sont pas tous soumis au même niveau d'exposition et de sensibilité aux IRA.

En effet, à l'échelle réduite des quartiers nous avons pu déterminer les principaux facteurs environnementaux responsables des IRA et qui varient d'un espace à l'autre.

La mise en relation cartographique et statistique des différents paramètres environnementaux avec les cas d'IRA rencontrés, nous a permis d'établir un lien entre les paramètres retenus et la sensibilité aux IRA. La démarche méthodologique et les outils utilisés pourraient être appliqués dans d'autres espaces aux caractéristiques similaires et utilisées comme un outil d'aide à la décision pour la réduction de l'incidence des IRA. 
Au total, toute intervention visant à améliorer l'état de santé des populations en ce qui concerne les IRA, doit cibler en priorité les quartiers Nord-est 2, Zone industrielle dans la commune de Koumassi et Vridi canal, Vridi 3 foyers dans celle de Port-Bouët, car ils sont les plus sensibles du fait de la proximité des industries.

\section{References:}

1. Airparif (2012). Surveillance de la qualité de l'air en Île-de-France : la pollution près du trafic. $\mathrm{N}^{\circ} 39,8 \mathrm{p}$.

2. Alla D.A. (2013). Risques naturels dans l'agglomération d'Abidjan (Côte d'Ivoire). Thèse d'Etat, Université Félix Houphouët Boigny, 385p.

3. Aubry P. (2014). Infections respiratoires aiguës. Médecine Tropicale, $7 p$.

4. Banai R. (1993). Fuzziness in geographical information systems : Contributions from the analytical hierarchy process. International Journal of Geographical Information Systems, 7 :315-329.

5. Branchu P., Badin A.L., Bechet B., Eisenlohr L., Le Priol T., Marseille F., Trielli E. (2013). Pollution d'origine routière et environnement de proximité. vertigO, hors-série 15 Février 2013, 58 p.

6. Chakhar S. (2006). Cartographie decisionnelle multicritere : formalisation et implementation informatique. Universite Paris Dauphine- Paris IX, 2006. Francais. <tel-00143960>

7. Dongo K.R., Niamke B.F., Adje A.F., Britton B.G.H., Nama L.A., Anoh K.P., Adima A.A., Atta K. (2013). Impacts des effluents liquides industriels sur l'environnement urbain d'Abidjan-Côte d'Ivoire. International journal of biological and chimical sciences. Vol 7, N 1, pp. 404-420.

8. Kouassi D., Kouamé F., Koné B., Biemi J., Tanner M., Cissé G. (2008). Analyse de la situation sanitaire des quartiers défavorisés dans le tissu urbain de Yopougon à Abidjan, Côte d'Ivoire. vertigO. volume 8, $\mathrm{N}^{\circ} 3,12 \mathrm{p}$.

9. Mesbah, S. (2009). Maladies infectieuses émergentes et réémergentes: le risque et la riposte en Algérie Médecine Tropicale 69 : 27-32.

10. Ministère de la santé et de la lutte contre le sida (2014). Rapport annuel sur la situation sanitaire 2013 en Côte d'Ivoire. 294p.

11. Ministère du plan et du développement (2012). Plan national de développement 2012-2015, tome III : vision de développement et orientations stratégiques, 132p. 
12. OMS (2011). Rapport annuel sur les statistiques sanitaires mondiales 2011, 170p.

13. OMS (2013). Rapport annuel sur la situation de la santé maternelle, néonatale et infantile en Afrique. 57p.

14. OMS (2013). Rapport annuel sur les statistiques sanitaires mondiales 2013, 172p.

15. Tchatchou D.N. (2012). Variations régionales de la survenance des infections respiratoires aiguës chez les enfants de moins de cinq ans au Cameroun. Mémoire de Master professionnel, Institut de Formation et de Recherche Démographiques de Yaoundé, 167p

16. TRAN A. (2004). Télédétection et épidémiologie : modélisation de la dynamique de populations d'insectes et application au contrôle de maladies à transmission vectorielle. Thèse de Doctorat, Université Louis Pasteur Strasbourg 1, 218p.

17. Woldemicael, G. (2000). The effects of water supply and sanitation on childhood mortality in urban Eritrea. Journal of Biosocial Sciences 32: 207-227.

18. Wu F. (1998). Simland : A prototype to simulate land conversion through the integrated GIS and CA with AHP-derived transition rules. International Journal of Geographical Information Science, 12 :63-82.

19. Zhu X. and Dale A.P. (2001). JavaAHP : a Web-based decision analysis tool for natural resource and environmental management. Environmental Modelling and Software, 16(3) :251-262. 